\title{
Population inversion in two-level systems possessing permanent dipoles
}

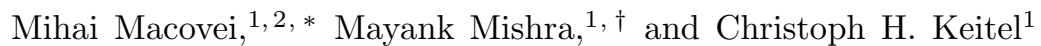 \\ ${ }^{1}$ Max-Planck-Institut für Kernphysik, Saupfercheckweg 1, D-69117 Heidelberg, Germany \\ ${ }^{2}$ Institute of Applied Physics, Academy of Sciences of Moldova, Academiei str. 5, MD-2028 Chişinău, Moldova
}

(Dated: April 15, 2021)

\begin{abstract}
Bare-state population inversion is demonstrated in a two-level system with all dipole matrix elements nonzero. A laser field is resonantly driving the sample whereas a second weaker and lower frequency coherent field additionally pumps it near resonance with the dynamically-Stark-splitted states. Due to existence of differing permanent dipole moments in the excited and ground bare states, quantum coherences among the involved dressed-states are induced leading to inversion in the steady-state. Furthermore, large refractive indices are feasible as well as the determination of the diagonal matrix elements via the absorption or emission spectra. The results apply to available biomolecular, spin or asymmetric quantum dot systems.

PACS numbers: $42.50 . \mathrm{Nn}, 87.15 .-\mathrm{v}, 78.67 . \mathrm{Hc}, 76.30 .-\mathrm{v}$
\end{abstract}

\section{INTRODUCTION}

Population inversion in a few-energy-level quantum system is strongly linked with its optical response and has resulted in traditional masing or lasing effects being successfully demonstrated [1 $\left[\begin{array}{l}3 \\ ]\end{array}\right.$. Subsequently, enormous economical and technological progress was triggered due to quantum electronics. However at present, atomic steady-state population inversion is achievable essentially only in pumped two-level atomic systems involving efforts via extra transitions with additional photon sources or environmental vacuum modifications [4]. Additionally, two-level quantum dot systems may also exhibit population inversion due to extra phonon induced decay rates [5]. Meanwhile, lasing without population inversion operates as well with the help of induced quantum coherences [6]. Moreover, free-electron laser sources involving inversions of momentum states exist at higher frequencies leading to original effects [7].

Over the past decade a range of experiments were performed demonstrating quantum effects in biological samples [8]. Particularly, energy transfer through quantum coherence in photosynthetic systems [9] was observed in [10, 11], while long-living quantum coherences survive in biological complexes even under normal conditions at room temperature [12]. Quantum coherence and entanglement in the processes of magneto-reception of the surrounding magnetic field [13, 14] were examined, too [15]. The single molecule fluorescence spectroscopy and the emitted photon quantum statistics are further excellent tools for research in quantum biology [16, 17]. Furthermore, coherent control of an effective two-level system in a non-Markovian biomolecular environment was investigated [18]. Additional quantum effects in biochemical systems are discussed, for instance, in [19]. An impor-

\footnotetext{
*Electronic address: macovei@phys.asm.md

${ }^{\dagger}$ Present address: IISER Mohali, Knowledge city, Sector 81, SAS Nagar, Manauli, Punjab, India, PO 140306.
}

tant issue raised in this context is to image tiny objects, such as biological cells or organic molecules and, therefore, highly refractive biological media are required [20].

Inspired by these remarkable advances in quantum biochemistry, here, we put forward a novel scheme that enables the creation of population inversion in certain biological samples acting as two-level systems with all possible dipole matrix elements being nonzero. The effect occurs due to induced quantum coherences which arise from the difference of the permanent dipole moments in the excited and ground states, respectively. This may allow for lasing or amplifying as well as optical switching devises in biomolecular materials. Large index of refractions without absorption are also feasible which may lead to an enhanced optical imaging resolution of the biomolecular sample due to a reduced probe-field wavelength inside the medium. Both the absorption or emission spectra can be used to extract the values of permanent dipoles.

The article is organized as follows. In Sec. II we describe the analytical approach and the system of interest, whereas in Sec. III we analyze the obtained results. A summary is given in Sec. IV.

\section{THEORETICAL FRAMEWORK}

We consider a two-level system possessing permanent dipoles and interacting with two external coherent laser fields. The first laser is near resonance with the transition frequency of the two-level sample while the second one is close to resonance with the dressed-frequency splitting due to the first laser, respectively (see Figure 1). As a concrete system, we may consider gammaglobulin macromolecules [19, 21] with the transition frequency $\omega_{21} \approx 4.8 \times 10^{15} \mathrm{~Hz}$, transition dipole moment $d \approx 1$ Debye and the difference between the diagonal dipole moments in the upper and lower bare states given by $\left|d_{22}-d_{11}\right| \approx 100$ Debye. However, the analytical formalism applies equally to spin [22], asymmetric semiconductor quantum dot [23] or other alternative [24] systems promising wider applications. The Hamiltonian describ- 


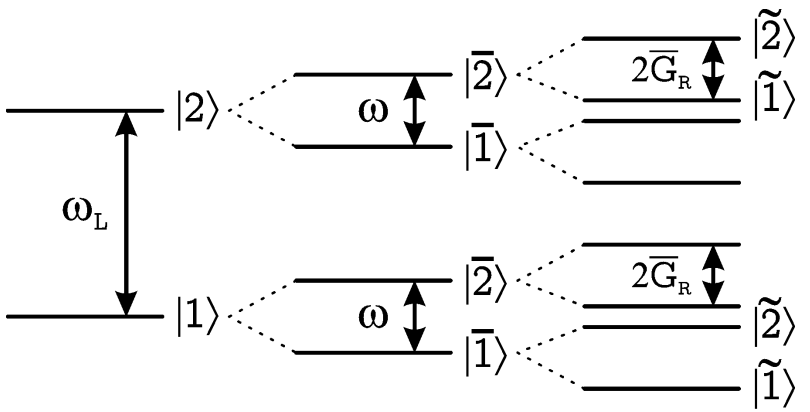

(a)

(b)

(c)

FIG. 1: Energy diagram of a two-level emitter with nonzero values of all involved dipole matrix elements $d_{\alpha \beta},\{\alpha, \beta=$ $1,2\}$. (a) A moderately intense laser field of frequency $\omega_{L}$ resonantly interacts with the molecular sample leading to dynamically Stark splitting of its energy levels. (b) A second coherent source of frequency $\omega$, close to generalized Rabi frequency due to first laser, is applied leading to transitions among the dressed-states. (c) The "double dressed-states" with $2 \bar{G}_{R}$ being the corresponding Rabi splitting frequency.

ing such a model, in a frame rotating at the first laser frequency $\omega_{L}$, and in the dipole approximation is:

$$
\begin{aligned}
H & =\sum_{k} \hbar\left(\omega_{k}-\omega_{L}\right) a_{k}^{\dagger} a_{k}+\hbar \Delta S_{z}+\hbar \Omega\left(S^{+}+S^{-}\right) \\
& +\hbar G S_{z} \cos (\omega t)+i \sum_{k}\left(\vec{g}_{k} \cdot \vec{d}\right)\left(a_{k}^{\dagger} S^{-}-a_{k} S^{+}\right) .
\end{aligned}
$$

In the Hamiltonian (1) the first three components are, respectively, the free energies of the environmental electromagnetic vacuum modes and molecular subsystems together with the laser-molecule interaction Hamiltonian. There, $\Omega=d E_{1} /(2 \hbar)$ is the corresponding Rabi frequency with $d \equiv d_{21}=d_{12}$ being the transition dipole moment while $E_{1}$ is the amplitude of the first laser field. The fourth term accounts for the second laser interacting at frequency $\omega$ and amplitude $E_{2}$ with the molecular system due to presence of permanent dipoles incorporated in $G$, i.e., $G=\left(d_{22}-d_{11}\right) E_{2} / \hbar$. The last term describes the interaction of the molecular subsystem with the environmental vacuum modes of the electromagnetic field reservoir. Further, $\vec{g}=\sqrt{2 \pi \hbar \omega_{k} / V} \vec{e}_{\lambda}$ is the molecule-vacuum coupling strength with $\vec{e}_{\lambda}$ being the photon polarization vector and $\lambda \in\{1,2\}$ whereas $V$ is the quantization volume. $\Delta=\omega_{21}-\omega_{L}$ is the laser field detuning from the molecular transition frequency $\omega_{21}$. The molecule barestate operators $S^{+}=|2\rangle\langle 1|$ and $S^{-}=\left[S^{+}\right]^{\dagger}$ obey the commutation relations $\left[S^{+}, S^{-}\right]=2 S_{z}$ and $\left[S_{z}, S^{ \pm}\right]=$ $\pm S^{ \pm}$. Here, $S_{z}=(|2\rangle\langle 2|-| 1\rangle\langle 1|) / 2$ is the bare-state inversion operator. $|2\rangle$ and $|1\rangle$ are, respectively, the excited and ground state of the molecule while $a_{k}^{\dagger}$ and $a_{k}$ are the creation and the annihilation operator of the $k_{t h}$ electromagnetic field mode, and satisfy the standard bosonic commutation relations, namely, $\left[a_{k}, a_{k^{\prime}}^{\dagger}\right]=\delta_{k k^{\prime}}$, and $\left[a_{k}, a_{k^{\prime}}\right]=\left[a_{k}^{\dagger}, a_{k^{\prime}}^{\dagger}\right]=0$ [25 28]. Notice that the
Hamiltonian (1) is incomplete. The following term

$$
\tilde{H}=\hbar \tilde{\Omega} S^{+} e^{i \omega_{L} t} \cos (\omega t)+\hbar \tilde{G} S_{z} \cos \left(\omega_{L} t\right) / 2+H . c .,
$$

will not be taken into account. Here, the first term describes the interaction of the second laser with the molecular system due to the transition dipole $d$, while the second one represents the interaction of the first laser with the molecule because of permanent dipoles and, hence, $\tilde{\Omega}=d E_{2} / \hbar$ whereas $\tilde{G}=\left(d_{22}-d_{11}\right) E_{1} / \hbar$. Since we assume realistic conditions that $\tilde{\Omega} \ll \omega_{L} \pm \omega$ [27, 28] as well as $\{\tilde{G}, \omega\} \ll \omega_{L}$ the Hamiltonian (2) can be considered as fast oscillating and, therefore, neglectable.

In the following, we shall consider a regime where the generalized Rabi frequency $\bar{\Omega}=\sqrt{\Omega^{2}+(\Delta / 2)^{2}}$ is larger than the single-molecule spontaneous decay rate as well as the coupling due to permanent dipoles, i.e. $\bar{\Omega} \gg \gamma$ and $\bar{\Omega}>G$. In this case it is more convenient to describe our system in the semi-classical laser-molecule dressed-state picture due to the first applied laser:

$$
|2\rangle=\cos \theta|\overline{2}\rangle-\sin \theta|\overline{1}\rangle \text { and }|1\rangle=\cos \theta|\overline{1}\rangle+\sin \theta|\overline{2}\rangle,
$$

with $\tan 2 \theta=2 \Omega / \Delta$. Here $|\overline{2}\rangle$ and $|\overline{1}\rangle$ are the corresponding upper and lower dressed states, respectively (see Figure 10). Applying the dressed-state transformation to the Hamiltonian (11) one arrives at the following Hamiltonian represented in a frame rotating also at the second laser field frequency, i.e. $\omega$,

$$
\begin{aligned}
H & =\sum_{k} \hbar\left(\omega_{k}-\omega_{L}\right) a_{k}^{\dagger} a_{k}+\hbar \bar{\Delta} R_{z}-\hbar \bar{G}\left(R^{+}+R^{-}\right) \\
& +i \sum_{k}\left(\vec{g}_{k} \cdot \vec{d}\right)\left\{a _ { k } ^ { \dagger } \left(\sin 2 \theta R_{z} / 2+\cos ^{2} \theta R^{-} e^{-i \omega t}\right.\right. \\
& \left.\left.-\sin ^{2} \theta R^{+} e^{i \omega t}\right)-H . c .\right\}
\end{aligned}
$$

where $\bar{\Delta}=\bar{\Omega}-\omega\left(1-\bar{G}^{2} / \omega^{2}\right) / 2 \equiv \bar{\Omega}-\omega / 2$ and $\bar{G}=$ $(G / 4) \sin 2 \theta$, and we have performed the rotating wave approximation with respect to $\omega$, i.e., we have assumed that $\omega \gg \bar{G}$. Eliminating the vacuum modes of the electromagnetic field reservoir in the usual way by adopting the Born-Markov approximations [25 28] one arrives then at the following dressed-state master equation:

$$
\begin{gathered}
\dot{\rho}(t)+i\left[\bar{\Delta} R_{z}-\bar{G}\left(R^{+}+R^{-}\right), \rho\right]=-\frac{\gamma_{0}}{4} \sin ^{2} 2 \theta\left[R_{z}, R_{z} \rho\right] \\
-\gamma_{+} \cos ^{4} \theta\left[R^{+}, R^{-} \rho\right]-\gamma_{-} \sin ^{4} \theta\left[R^{-}, R^{+} \rho\right]+H . c .
\end{gathered}
$$

Here $\gamma_{0, \pm}$ are the single-qubit spontaneous decay rates corresponding to dressed-state frequencies $\omega_{L}$ and $\omega_{L} \pm$ $2 \bar{\Omega}$, respectively. The new quasi-spin operators, i.e., $R^{+}=|\overline{2}\rangle\langle\overline{1}|, R^{-}=\left[R^{+}\right]^{\dagger}$ and $R_{z}=|\overline{2}\rangle\langle\overline{2}|-| \overline{1}\rangle\langle\overline{1}|$ are operating in the dressed-state picture. They obey the following commutation relations: $\left[R^{+}, R^{-}\right]=R_{z}$ and $\left[R_{z}, R^{ \pm}\right]= \pm 2 R^{ \pm}$. Notice that in the above master equation, we have neglected the rapidly oscillating terms in the spontaneous emission part - an approximation valid when $\{\bar{\Omega}, \omega\} \gg\left\{\gamma_{0}, \gamma_{ \pm}\right\}$.

In the following section, we will discuss our results, i.e., the possibility to create bare-state population inversion as well as high refractive media. 

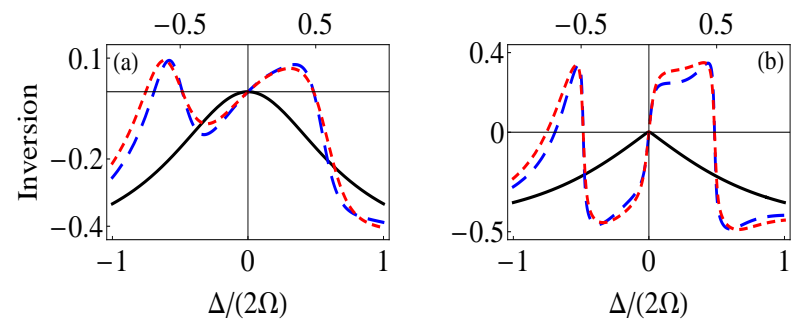

FIG. 2: (color online) The steady-state dependence of the bare-state inversion operator $\left\langle S_{z}\right\rangle / N$ versus the scaled parameter $\Delta /(2 \Omega)$. The solid line is plotted for $G / \gamma=0$, the longdashed one is for $G / \gamma=16$ while the short-dashed curve corresponds to $G / \gamma=24$. Other parameters are: $\Omega /(N \gamma)=45$ and $\omega /(N \gamma)=100$ with $\gamma \approx 2.6 \mathrm{MHz}$ as feasible for gammaglobulin. (a) $N=1$ whereas (b) $N=50$ while molecules are dense enough to allow for collectivity.

\section{RESULTS AND DISCUSSION}

One can observe from Eq. (5) that permanent dipoles lead to the appearance of a pumping term among the dressed states. This term contributes to a completely unexpected behavior of the two-level system. In particular, as we shall see, it can induce pumping the system into an inverted state. The system of equations for the dressedstate inversion and dressed-state polarization operators can be obtained from the master equation (5), namely,

$$
\begin{aligned}
\left\langle\dot{R}_{z}\right\rangle & =-2 i \bar{G}\left(\left\langle R^{-}\right\rangle-\left\langle R^{+}\right\rangle\right)-2 \Gamma_{+}\left\langle R_{z}\right\rangle+2 \Gamma_{-}, \\
\left\langle\dot{R}^{+}\right\rangle & =(2 i \bar{\Delta}-\Gamma)\left\langle R^{+}\right\rangle+i \bar{G}\left\langle R_{z}\right\rangle,
\end{aligned}
$$

with $\left\langle R^{-}\right\rangle=\left[\left\langle R^{+}\right\rangle\right]^{\dagger}$. Here, $\Gamma_{ \pm}=\gamma\left(\sin ^{4} \theta \pm \cos ^{4} \theta\right), \Gamma=$ $\Gamma_{+}+\gamma \sin ^{2} 2 \theta$ and we have considered that $\gamma_{0}=\gamma_{ \pm} \equiv$ $\gamma$ which is the case for a free-space setup. The meanvalue of the bare-state inversion operator $\left\langle S_{z}\right\rangle$ can be represented via dressed-state operators as follows:

$$
\left\langle S_{z}\right\rangle=\cos 2 \theta\left\langle R_{z}\right\rangle / 2-\sin 2 \theta\left(\left\langle R^{+}\right\rangle+\left\langle R^{-}\right\rangle\right) / 2 .
$$

From the system of equations (6) one immediately obtains the steady-state relations:

$$
\left\langle R^{+}\right\rangle=i \bar{G}\left\langle R_{z}\right\rangle /(\Gamma-2 i \bar{\Delta}) \text { and }\left\langle R^{-}\right\rangle=\left[\left\langle R^{+}\right\rangle\right]^{\dagger} .
$$

Inserting (8) in Eq. (7) one arrives at:

$$
\left\langle S_{z}\right\rangle=\left(\cos 2 \theta+4 \bar{\Delta} \bar{G} \sin 2 \theta /\left[\Gamma^{2}+(2 \bar{\Delta})^{2}\right]\right)\left\langle R_{z}\right\rangle / 2,
$$

where, again, from Eqs. (6) one has that:

$$
\left\langle R_{z}\right\rangle=2 \Gamma_{-} /\left[2 \Gamma_{+}+(2 \bar{G})^{2} \Gamma /\left(\Gamma^{2}+(2 \bar{\Delta})^{2}\right)\right] .
$$

An interesting result here is the non-zero value for the dressed-state coherences $\left\langle R^{ \pm}\right\rangle$, see Eq. (8). In the absence of permanent dipoles, i.e. $G=0$, these quantities are zero in the moderately intense pumping regime considered here. Therefore, new physics is expected due to existence of permanent dipoles in bichromatic pumping fields. In particular, Figure2(a) shows the mean-value of the single-molecule bare-state inversion operator for particular parameters of interest. Steady-state inversion in the bare states is achieved when $\left\langle S_{z}\right\rangle>0$ and it occurs in the presence of permanent dipoles. On the other hand, Figure 3(a) depicts the real part of the mean-value of the dressed-state coherence operator $\left\langle R^{+}\right\rangle$in steady-state. The minima observed in these behaviors correspond to an inverted molecular bare-state system (compare Fig. 2a and Fig. 3a). Thus, inversion occurs due to the real part of the dressed-state coherences which can be nonzero in our system.

One can apply the double dressed-state formalism (see Figure 1) in order to obtain further information on our system, namely,

$$
|\overline{2}\rangle=\cos \phi|\tilde{2}\rangle+\sin \phi|\tilde{1}\rangle,|\overline{1}\rangle=\cos \phi|\tilde{1}\rangle-\sin \phi|\tilde{2}\rangle .
$$

This approach is particularly useful to account for vacuum induced collective effects among the emitters and their corresponding influences on molecular dynamics. Introducing (11) in the Hamiltonian (4) and, again, eliminating the degrees of freedom related with the environmental vacuum modes in the Born-Markov approximations one arrives at the double dressed master equation:

$$
\begin{aligned}
\dot{\rho}(t) & +i \bar{G}_{R}\left[\tilde{R}_{z}, \rho\right]=-\bar{\Gamma}_{0}\left[\tilde{R}_{z}, \tilde{R}_{z} \rho\right]-\bar{\Gamma}_{+}\left[\tilde{R}^{+}, \tilde{R}^{-} \rho\right] \\
& -\bar{\Gamma}_{-}\left[\tilde{R}^{-}, \tilde{R}^{+} \rho\right]+H . c .
\end{aligned}
$$

Here, $\bar{\Gamma}_{0}=\gamma\left(\omega_{L}\right) \sin ^{2} 2 \theta \cos ^{2} 2 \phi / 4+\sin ^{2} 2 \phi\left\{\gamma\left(\omega_{L}+\right.\right.$ $\left.\omega) \cos ^{4} \theta+\gamma\left(\omega_{L}-\omega\right) \sin ^{4} \theta\right\} / 4, \quad \bar{\Gamma}_{+}=\gamma\left(\omega_{L}+\right.$ $\left.2 \bar{G}_{R}\right) \sin ^{2} 2 \phi \sin ^{2} 2 \theta / 4+\gamma\left(\omega_{L}+\omega+2 \bar{G}_{R}\right) \cos ^{4} \phi \cos ^{4} \theta+$ $\gamma\left(\omega_{L}-\omega+2 \bar{G}_{R}\right) \sin ^{4} \theta \sin ^{4} \phi$ and $\bar{\Gamma}_{-}=\gamma\left(\omega_{L}-\right.$ $\left.2 \bar{G}_{R}\right) \sin ^{2} 2 \phi \sin ^{2} 2 \theta / 4+\gamma\left(\omega_{L}+\omega-2 \bar{G}_{R}\right) \cos ^{4} \theta \sin ^{4} \phi+$ $\gamma\left(\omega_{L}-\omega-2 \bar{G}_{R}\right) \sin ^{4} \theta \cos ^{4} \phi$ with $\cot 2 \phi=\bar{\Delta} / \bar{G}$, and $\bar{G}_{R}=\sqrt{\Delta^{2}+\bar{G}^{2}}$. The new operators, i.e., $\tilde{R}^{+}=$ $|\tilde{2}\rangle\langle\tilde{1}|, \quad \tilde{R}^{-}=\left[\tilde{R}^{+}\right]^{\dagger}$ and $\tilde{R}_{z}=|\tilde{2}\rangle\langle\tilde{2}|-| \tilde{1}\rangle\langle\tilde{1}|$ are operating in the double dressed-state picture obeying the following commutation relations: $\left[\tilde{R}^{+}, \tilde{R}^{-}\right]=\tilde{R}_{z}$ and $\left[\tilde{R}_{z}, \tilde{R}^{ \pm}\right]= \pm 2 \tilde{R}^{ \pm}$. The master equation (12) contains only slowly varying terms in the spontaneous emission
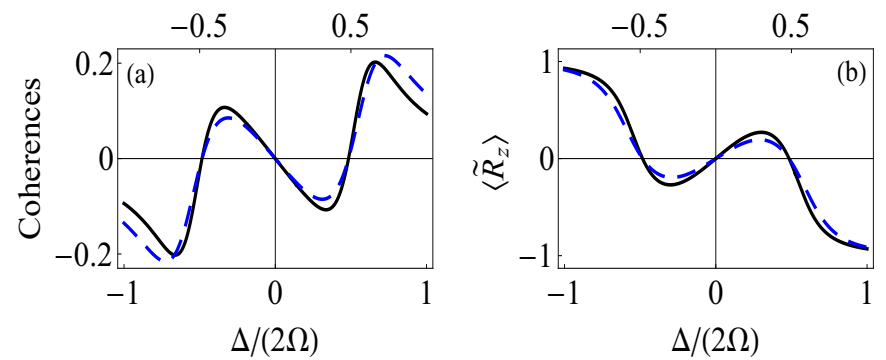

FIG. 3: (color online) The single-molecule steady-state behaviors of (a) the real part of the dressed-state coherences $\left\langle R^{+}\right\rangle$ and (b) the double dressed-state inversion operator $\left\langle\tilde{R}_{z}\right\rangle$ versus the scaled detuning $\Delta /(2 \Omega)$. The solid line is plotted for $G / \gamma=16$ while the dashed curve corresponds to $G / \gamma=24$. Here $\Omega / \gamma=45$ and $\omega / \gamma=100$. 
damping, that is, we have assumed that $\bar{G}_{R} \gg \gamma(\tilde{\omega})$, with $\gamma(\tilde{\omega})=2 d^{2} \tilde{\omega}^{3} /\left(3 \hbar c^{3}\right)$ being the single-molecule spontaneous decay rate corresponding to the double dressedstate frequency $\tilde{\omega}$ (see Figure 1).

The steady-state solution of Eq. (12) can be chosen in the form:

$$
\rho=Z^{-1} \exp \left[-\eta \tilde{R}_{z}\right]
$$

where the normalization $Z$ is determined from the relation $\operatorname{Tr}\{\rho\}=1$. Inserting (13) into (12) one obtains $\eta=\ln \left(\bar{\Gamma}_{+} / \bar{\Gamma}_{-}\right) / 2$. Using the relations (17) and (11) we arrive at the following expression for the mean value of the bare-state inversion operator $\left\langle S_{z}\right\rangle$ represented via the double dressed-state inversion operator $\left\langle\tilde{R}_{z}\right\rangle$, respectively,

$$
\left\langle S_{z}\right\rangle=\cos [2(\theta-\phi)]\left\langle\tilde{R}_{z}\right\rangle / 2 .
$$

The steady-state expression for the double dressed-state inversion operator can be obtained with the help of Eq. (13) and the coherent molecular state $|s\rangle \equiv|N-s, s\rangle$ which denotes a symmetrized $N$-molecule state in which $N-s$ molecules are in the lower double dressed-state $|\tilde{1}\rangle$ and $s$ molecules are excited to the upper double dressedstate $|\tilde{2}\rangle$, respectively [27]. Thus,

$$
\left\langle\tilde{R}_{z}\right\rangle=-N+\frac{\left(x^{1+N}-1\right)-(1+N)(x-1)}{2^{-1}(x-1)\left(x^{1+N}-1\right)},
$$

where $x=\bar{\Gamma}_{+} / \bar{\Gamma}_{-}$. In this case the molecular system has to occupy a volume with linear dimensions of the order of the smallest relevant emission wavelength or less. However, this restriction may be relaxed for certain geometries 25 28]. The molecular operators entering in Eqs. (12 15) are then collective ones, i.e. $\tilde{R}^{ \pm}=\sum_{j=1}^{N} \tilde{R}_{j}^{ \pm}$ as well as $\tilde{R}_{z}=\sum_{j=1}^{N} \tilde{R}_{z j}$. Figure 2 (b) shows the bare-state inversion operator $\left\langle S_{z}\right\rangle / N$, based on the expressions (14) and (15), for a collection of $N=50$ molecules in a volume $\left(2 \pi c / \omega_{21}\right)^{3}$ or a molecular density $\bar{N} \approx 7.8 \times 10^{14} \mathrm{~cm}^{-3}$. Almost full inversion in
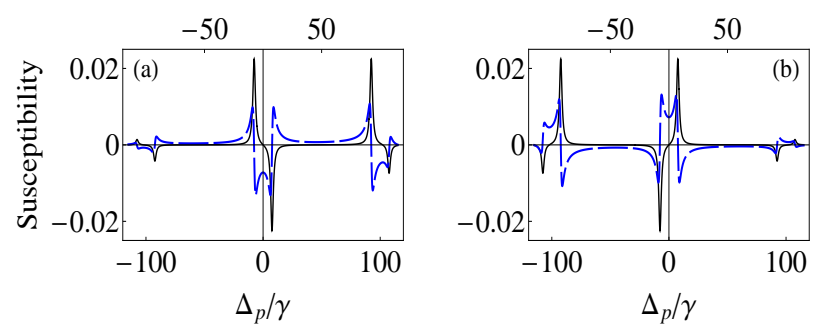

FIG. 4: (color online) The steady-state dependence of the linear susceptibility $\chi\left(\nu_{p}\right)$ [in units of $\bar{N} d^{2} /(\hbar \gamma)$ ] versus scaled detuning $\Delta_{p} / \gamma$. The solid black curve corresponds to the imaginary part (absorption spectrum) while the long-dashed blue line to the real part of the susceptibility, respectively. (a) $\Delta /(2 \Omega)=0.43$ whereas (b) $\Delta /(2 \Omega)=-0.43$. Other parameters are: $\Omega / \gamma=45, \omega / \gamma=100, G / \gamma=16$. the steady-state is achieved when we consider that the double-dressed decay rates $\gamma(\tilde{\omega})$ are equal and denoted by $\gamma$. Furthermore, abrupt population behaviors are observed as well which may be used for engineering optical switching devices with switching times of the order of $(N \gamma)^{-1}$. Note that the mean values of non-diagonal terms resulting from Eq. (12) are zero in the steady-state.

We proceed by calculating the refractive properties of a very weak field probing the strongly driven molecular sample around the first laser's frequency $\omega_{L}$. The linear susceptibility $\chi(\nu)$ of the probe field, at frequency $\nu$, can be represented in terms of the Fourier transform of the average value of the two-time commutator of the molecular operator as

$$
\chi(\nu)=\frac{i}{\hbar} \bar{N} d^{2} \int_{0}^{\infty} d \tau e^{i\left(\nu-\omega_{L}\right) \tau}\left\langle\left[S^{-}(\tau), S^{+}\right]\right\rangle_{s} .
$$

Note that the subindex $s$ means steady-state. Inserting the dressed-state transformations (31) and (11) in (16), in a frame rotating also at the second laser's frequency $\omega$, and using the master equation (12) one arrives at the following expression for the susceptibility, namely,

$$
\begin{aligned}
& \chi(\nu)=\frac{i}{\hbar} \bar{N} d^{2}\left\langle\tilde{R}_{z}\right\rangle\left\{\frac { 1 } { 4 } \operatorname { s i n } ^ { 2 } 2 \theta \operatorname { s i n } ^ { 2 } 2 \phi \left(\chi_{1}\left(\Delta_{p}, 2 \bar{G}_{R}\right)\right.\right. \\
- & \left.\chi_{1}\left(\Delta_{p},-2 \bar{G}_{R}\right)\right)+\cos ^{4} \theta\left(\sin ^{4} \phi \chi_{2}\left(\Delta_{p}, 2 \bar{G}_{R},-\omega\right)\right. \\
- & \left.\cos ^{4} \phi \chi_{2}\left(\Delta_{p},-2 \bar{G}_{R},-\omega\right)\right)+\sin ^{4} \theta\left(\cos ^{4} \phi\right. \\
\times & \left.\left.\chi_{3}\left(\Delta_{p}, 2 \bar{G}_{R}, \omega\right)-\sin ^{4} \phi \chi_{3}\left(\Delta_{p},-2 \bar{G}_{R}, \omega\right)\right)\right\},
\end{aligned}
$$

where $\chi_{1}\left(\Delta_{p}, x\right)=\left(\bar{\Gamma}_{s}+i\left(\Delta_{p}+x\right)\right) /\left[\bar{\Gamma}_{s}^{2}+\left(\Delta_{p}+x\right)^{2}\right]$, $\chi_{2}\left(\Delta_{p}, x,-y\right)=\left(\bar{\Gamma}_{s}+i\left(\Delta_{p}+x-y\right)\right) /\left[\bar{\Gamma}_{s}^{2}+\left(\Delta_{p}+x-y\right)^{2}\right]$ and $\chi_{3}\left(\Delta_{p}, x, y\right)=\left(\bar{\Gamma}_{s}+i\left(\Delta_{p}+x+y\right)\right) /\left[\bar{\Gamma}_{s}^{2}+\left(\Delta_{p}+x+y\right)^{2}\right]$ whereas $\Delta_{p}=\nu-\omega_{L}$ while $\bar{\Gamma}_{s}=4 \bar{\Gamma}_{0}+\bar{\Gamma}_{+}+\bar{\Gamma}_{-}$. Figure (4) shows the steady-state behavior of the linear susceptibility when the molecular sample is probed with a weak coherent field of frequency $\nu$. Both, positive or negative dispersions without absorption are clearly visible around $\Delta_{p} / \gamma=0$ which may lead to enhanced or reduced refractive indices applicable for optical imaging, lithography or negative refraction processes in dense media [29]. In particular, the index of refraction close to vanishing absorption $n(\nu) \approx \sqrt{1+\chi^{\prime}}$ takes values $n>2$ for $\bar{N}=10^{17} \mathrm{~cm}^{-3}$ and $\Delta /(2 \Omega)=-0.43$. These dependences for the susceptibility $\chi(\nu)$ are easily understood in the double dressed-state picture (see Fig. 1r). Particularly, for $\Delta /(2 \Omega)=0.43$ there are more molecules in the upper double dressed state $|\tilde{2}\rangle$, i.e. $\left\langle\tilde{R}_{z}\right\rangle>0$ (see Fig. [3b). As a consequence, at frequency $\omega_{L}+\omega+2 \bar{G}_{R}$ one has gain while at frequency $\omega_{L}-\omega-2 \bar{G}_{R}$ we have absorption, see the lateral dip/peak in Figure (4ha). Similarly one can explain the whole structure shown in Figure $4(\mathrm{a}, \mathrm{b})$. An interesting issue here is the structure at $\omega_{L} \pm 2 \bar{G}_{R}$ which may help to extract the value of permanent dipoles, i.e. $\left|d_{22}-d_{11}\right|$. If one inspects the absorption spectrum shown in Fig. 4 then the frequency separation between the first maximum and minimum around $\Delta_{p}=0$ is equal to $4 \bar{G}_{R} \cdot \bar{G}_{R}$ involves the difference of 
permanent dipoles $\left|d_{22}-d_{11}\right|$. This, in principle, allows to determine $\left|d_{22}-d_{11}\right|$ if all other involved parameters are known. Finally, the elastic photon scattering spectrum consists of three lines at $\left\{\omega_{L}, \omega_{L} \pm \omega\right\}$. The inelastic one may contain up to nine spectral lines in strict concordance with the double dressed-state formalism schematically shown in Fig. (15). Suppression of a spectral line at the frequency of the strongly driving laser also occurs [30]. As well, the emission spectrum permits determination of the diagonal dipole matrix elements (see also Ref. [31]). The magnitudes of permanent dipoles are required for e.g. interpretation of biological images as well as for biological high-harmonic or ultrashort pulse generation processes via laser pumped media possessing permanent dipoles 32] (see also [33]).

\section{SUMMARY}

Summarizing, we have investigated the steady-state quantum dynamics of laser pumped two-level molecular samples with broken inversion symmetry. We have demonstrated population inversion in the bare states due to induced coherences which in turn depend on the magnitude of permanent dipoles. Vacuum mediated collective effects among the two-level emitters considerably enhance the molecular inversion. The values of the permanent dipoles can be inferred from the emission or absorption spectra. Furthermore, the investigated system exhibits large positive or negative dispersion without absorption facilitating applications including optical imaging, lithography and negative indices of refraction. The results apply especially to biomolecular, spin or asymmetrical quantum dot systems.

\section{Acknowledgments}

M.M. and C.H.K. acknowledge the financial support by the German Federal Ministry of Education and Research, grant No. 01DK13015, and Academy of Sciences of Moldova, grant No. 13.820.05.07/GF. M.M. and M.M. are grateful for the nice hospitality of the Theory Division of the Max Planck Institute for Nuclear Physics from Heidelberg, Germany.
[1] A. L. Schawlow and C. H. Townes, Phys. Rev. 112, 1940 (1958)

[2] A. M. Prokhorov, JETP 34, 1656 (1958).

[3] N. G. Basov, O. N. Krokhin, and Yu. M. Popov, Sov. Phys. Usp. 3, 702 (1961).

[4] C. M. Savage, Phys. Rev. Lett. 60, 1828 (1988); M. H. Anderson, G. Vemuri, J. Cooper, P. Zoller and S. J. Smith, Phys. Rev. A 47, 3202 (1993). S. John and T. Quang, Phys. Rev. Lett. 78, 1888 (1997); S. Hughes and H. J. Carmichael, ibid. 107, 193601 (2011); T. Quang and H. Freedhoff, Phys. Rev. A 47, 2285 (1993).

[5] T. M. Stace, A. C. Doherty and S. D. Barrett, Phys. Rev. Lett. 95, 106801 (2005); M. Glässl, A. M. Barth and V. M. Axt, ibid. 110, 147401 (2013); S. Das and M. A. Macovei, Phys. Rev. B 88, 125306 (2013); J. H. Quilter, A. J. Brash, F. Liu, M. Glässl, A. M. Barth, V. M. Axt, A. J. Ramsay, M. S. Skolnick and A. M. Fox, arXiv:1409.0913v3 [cond-mat.mes-hall].

[6] O. A. Kocharovskaya and Ya. I. Khanin, JETP Lett. 48, 630 (1988); S. E. Harris, Phys. Rev. Lett. 62, 1033 (1989); M. O. Scully, S.-Y. Zhu and A. Gavrielides, ibid. 62, 2813 (1989).

[7] K. P. Heeg, H.-Ch. Wille, K. Schlage, T. Guryeva, D. Schumacher, I. Uschmann, K. S. Schulze, B. Marx, T. Kämpfer, G. G. Paulus, R. Röhlsberger and J. Evers, Phys. Rev. Lett. 111, 073601 (2013); K. P. Heeg, J. Haber, D. Schumacher, L. Bocklage, H.-Ch. Wille, K. S. Schulze, R. Loetzsch, I. Uschmann, G. G. Paulus, R. Rüffer, R. Röhlsberger, and J. Evers, ibid. 114, 203601 (2015); K.P. Heeg, C. Ott, D. Schumacher, H.-C. Wille, R. Röhlsberger, T. Pfeifer, and J. Evers, ibid. 114, 207401 (2015).

[8] N. Lambert, Y.-N. Chen, Y.-C. Cheng, C.-M. Li, G.-Y. Chen and F. Nori, Nature Phys. 9, 10 (2013).
[9] G. S. Engel, Procedia Chemistry 3, 222 (2011).

[10] G. S. Engel, T. R. Calhoun, E. L. Read, T.-K. Ahn, T. Mancal, Y.-C. Cheng, R. E. Blankenship and G. R. Fleming, Nature (London) 446, 782 (2007).

[11] R. Hildner, D. Brinks, J. B. Nieder, R. J. Cogdell and N. F. van Hulst, Science 340, 1448 (2013).

[12] E. Collini and G. D. Scholes, Science 323, 369 (2009).

[13] T. Ritz, P. Thalau, J.B. Phillips, R. Wiltschko and W. Wiltschko, Nature (London) 429, 177 (2004).

[14] J. Cai and M. B. Plenio, Phys. Rev. Lett. 111, 230503 (2013).

[15] E. M. Gauger, E. Rieper, J. J. L. Morton, S. C. Benjamin and V. Vedral, Phys. Rev. Lett. 106, 040503 (2011); V. Vedral, Procedia Chemistry 3, 172 (2011).

[16] H. P. Lu, L. Xun and X. S. Xie, Science 282, 1877 (1998).

[17] Y. Zheng and F. L. H. Brown, Phys. Rev. Lett. 90, 238305 (2003).

[18] J. Eckel, J. H. Reina and M. Thorwart, New Jr. of Phys. 11, 085001 (2009).

[19] V. A. Kovarskii, Phys. Usp. 42, 797 (1999).

[20] M. O. Scully, Phys. Rev. Lett. 67, 1855 (1991); Ch. O'Brien, P. M. Anisimov, Yu. Rostovtsev, and O. Kocharovskaya, Phys. Rev. A 84, 063835 (2011).

[21] R. B. Setlow, E. C. Pollard, Molecular Biophysics (Oxford: Pergamon Press, 1962).

[22] A. S. M. Windsor, C. Wei, S. A. Holmstrom, J. P. D. Martin and N. B. Manson, Phys. Rev. Lett. 80, 3045 (1998).

[23] O. V. Kibis, G. Ya. Slepyan, S. A. Maksimenko and A. Hoffmann, Phys. Rev. Lett. 102, 023601 (2009); F. Oster, C. H. Keitel and M. Macovei, Phys. Rev. A 85, 063814 (2012); E. Paspalakis, J. Boviatsis and S. Baskoutas, J. Appl. Phys. 114, 153107 (2013).

[24] M. Terauchi, and T. Kobayashi, Chem. Phys. Lett. 137, 
319 (1987); A. Brown, and W. J. Meath, J. Chem. Phys. 109, 9351 (1998); Ch. Brunel, B. Lounis, Ph. Tamarat, and M. Orrit, Phys. Rev. Lett. 81, 2679 (1998); V. Puller, B. Lounis, and F. Pistolesi, ibid. 110, 125501 (2013); W. Yang, Sh. Gong, and Zh. Xu, Opt. Express 14, 7216 (2006); F. Herrera, B. Peropadre, L. A. Pachon, S. K. Saikin, A. Aspuru-Guzik, arXiv:1409.1930v1 [quant-ph].

[25] G. S. Agarwal, Quantum Statistical Theories of Spontaneous Emission and their Relation to other Approaches (Springer, Berlin, 1974).

[26] L. Allen, J. H. Eberly, Optical Resonance and Two-Level Atoms (Dover, New York, 1975).

[27] R. R. Puri, Mathematical Methods of Quantum Optics (Springer, Berlin 2001).

[28] M. Kiffner, M. Macovei, J. Evers, and C. H. Keitel,
Progress in Optics 55, 85 (2010).

[29] N. A. Proite, B. E. Unks, J. T. Green, and D. D. Yavuz, Phys. Rev. Lett. 101, 147401 (2008); Z. J. Simmons, N. A. Proite, J. Miles, D. E. Sikes, and D. D. Yavuz, Phys. Rev. A 85, 053810 (2012).

[30] Y. He et al., Phys. Rev. Lett. 114, 097402 (2015).

[31] O. Postavaru, Z. Harman, and C. H. Keitel, Phys. Rev. Lett. 106, 033001 (2011).

[32] V. A. Kovarsky, B. S. Philipp, and E. V. Kovarsky, Phys. Lett. A 226, 321 (1997); W. Yang, Sh. Gong, R. Li, and Zh. Xu, ibid. 362, 37 (2007).

[33] J. Deiglmayr, A. Grochola, M. Repp, O. Dulieu, R. Wester, and M. Weidemüller, Phys. Rev. A 82, 032503 (2010). 